\title{
MultiTACT: Uma abordagem para a construção de atividades de ensino multidisciplinares para estimular o Pensamento Computacional no Ensino Fundamental I
}

\author{
Suéllen Rodolfo Martinelli ${ }^{1}$, Luciana Aparecida Martinez Zaina ${ }^{1}$, \\ Tiemi Christine Sakata ${ }^{1}$ \\ ${ }^{1}$ Departamento de Computação de Sorocaba (DComp-So) \\ Universidade Federal de São Carlos (UFSCar) - Sorocaba - SP - Brasil \\ suellen.r.martinelli@gmail.com, \{lzaina,tiemi\}@ufscar.br
}

\begin{abstract}
Computational Thinking (CT) can be promoted by applying multidisciplinary activities and using an unplugged approach. This paper presents an approach, named MultiTACT Approach, that enables teachers to build and to apply teaching activities related to CT. These activities aim to foster the skills of logic, abstraction, decomposition in Primary School students. The approach was conceived from a qualitative analysis of 51 teaching activities that were obtained from three editions of a continuing education course. We identified a set of teaching strategies that are aligned with recommendations found in the Common National Curriculum Base.
\end{abstract}

Resumo. O Pensamento Computacional $(P C)$ pode ser promovido por meio da aplicação de atividades multidisciplinares e de práticas desplugadas. Este artigo apresenta uma abordagem, chamada de Abordagem MultiTACT, que possibilita aos professores construir e aplicar atividades de ensino relacionadas ao PC. Essas práticas visam fomentar habilidades de lógica, abstração, decomposição em estudantes do Ensino Fundamental I. A abordagem foi concebida a partir de uma análise qualitativa de 51 práticas que foram obtidas em três cursos de formação continuada ministrados. Foram detectados um conjunto de estratégias de ensino que são alinhadas com as recomendações dadas pela Base Nacional Comum Curricular.

\section{Introdução e Motivação}

O Pensamento Computacional (PC) ou Computational Thinking foi difundido na academia a partir de 2006. Trata-se do emprego de "ferramentas mentais", como a capacidade de se pensar de forma abstrata, reduzir problemas em partes menores e gerenciáveis com a finalidade de resolver problemas [Wing 2006]. Ele não se restringe à problemáticas da Computação e possibilita as crianças serem mais inventivas e criativas [Mohaghegh and Mccauley 2016].

O desenvolvimento do PC no Ensino Básico já é uma realidade no continente europeu. No Brasil, o PC é caracterizado pela Base Nacional Comum Curricular (BNCC) como uma competência da área de Matemática e atrelada à Cultura Digital [Valente et al. 2017]. O desafio é como viabilizar a apropriação desse "pensar computacional" entre docentes que atuam em escolas com pouca ou nenhuma infraestrutura, 
VIII Congresso Brasileiro de Informática na Educação (CBIE 2019)

Anais dos Workshops do VIII Congresso Brasileiro de Informática na Educação (WCBIE 2019)

uma vez que $65 \%$ delas não oferecem laboratórios de informática e outras $44,1 \%$ não possuem Internet [INEP 2019]. É preciso considerar as necessidades de cada instituição, sem gastos financeiros excessivos. Isso implica em tornar acessível o conhecimento sobre estratégias de ensino que promovam o PC nas práticas educacionais [Valente et al. 2017].

Este artigo apresenta a Abordagem MultiTACT - Atividades de Ensino de Múltiplas Facetas para o desenvolvimento do Pensamento Computacional (do inglês, Multi-facets Teaching Activities for developing Computational Thinking Approach). Ela esclarece uma técnica procedural e um conjunto de recomendações que visa auxiliar o docente na concepção e mediação de atividades de ensino que estimulem o PC entre crianças do Ensino Fundamental I (EFI). A proposta emergiu da análise qualitativa de práticas dedicadas ao fomento do PC, sendo estas obtidas durante três formações continuadas ministradas.

\section{Estado da Arte}

Apesar do PC ser um termo comum à comunidade da Computação, há confusão entre docentes sobre o seu significado, sendo equivocadamente comparado ao uso de tecnologias na educação [Nicastro et al. 2018]. Por não existir um consenso na literatura, este estudo baseou-se em organizações como a Computer Science Teachers Association (CSTA), a International Society for Technology in Education (ISTE), a Association for Computing Machinery (ACM) e a Sociedade Brasileira de Computação [SBC 2018].

Este estudo considera as habilidades do PC como: Raciocínio Lógico, para analisar fatos e estruturar ideias sobre um problema; Algoritmos / Procedimentos, gerar uma sequência finita de instruções para resolver uma tarefa; Decomposição / Generalização, quebrar um problema em partes ou analisar partes de um todo; Reconhecer Padrões, identificar similaridades entre problemas e aplicá-las em situações semelhantes; Abstração, sintetizar fatos para decidir o que é importante e ignorar detalhes; Paralelismo, capacidade em distribuir recursos para realizar tarefas simultâneas; e Manipulação de Dados, coletar, avaliar e representar dados de forma sistêmica [Martinelli and Sakata 2018].

Existem abordagens e frameworks na literatura com o objetivo de auxiliar o docente na articulação do PC em sala de aula. Elas têm foco no Ensino Médio (EM) e no envolvimento dos alunos com problemas que associam o PC à disciplinas da área de exatas, sendo necessário propor soluções fixadas em linguagens de programação [Sengupta et al. 2013, Seiter and Foreman 2013]. No Brasil, também encontram-se algumas abordagens. $\mathrm{O}$ modelo $P e n C$ tem características próximas às abordagens citadas, por depender de ambientes de programação e ser para o EM [França and Tedesco 2015]. Outras propostas apontam ações a serem adotadas para a elaboração de práticas que estimulem o PC. Destas, [Nicastro et al. 2018] apresenta um método para professores do EFI, enquanto o framework MUDDI aplica-se ao Ensino Infantil [Gomes 2018].

Este trabalho difere dos demais por expor uma proposta que fornece processos e recomendações para conceber, documentar e conduzir práticas sobre PC. Mesmo com dados processos, a Abordagem MultiTACT dá flexibilidade para usar diferentes estratégias de ensino, recursos e materiais, não limitando o professor no emprego de recursos digitais. Outro diferencial é a opção de construir atividades multidisciplinares a partir da definição de problemas que estimulam habilidades da BNCC junto ao PC. Desse modo, as práticas desenvolvidas a partir da Abordagem MultiTACT coloca o aluno diante de problemas que, para serem solucionados, dependem da aplicabilidade de habilidades e competências 
VIII Congresso Brasileiro de Informática na Educação (CBIE 2019)

Anais dos Workshops do VIII Congresso Brasileiro de Informática na Educação (WCBIE 2019)

pertinentes ao PC e aos demais componentes curriculares.

\section{Metodologia}

A metodologia de condução deste trabalho é ilustrada pela Figura 1 e descrita a seguir.

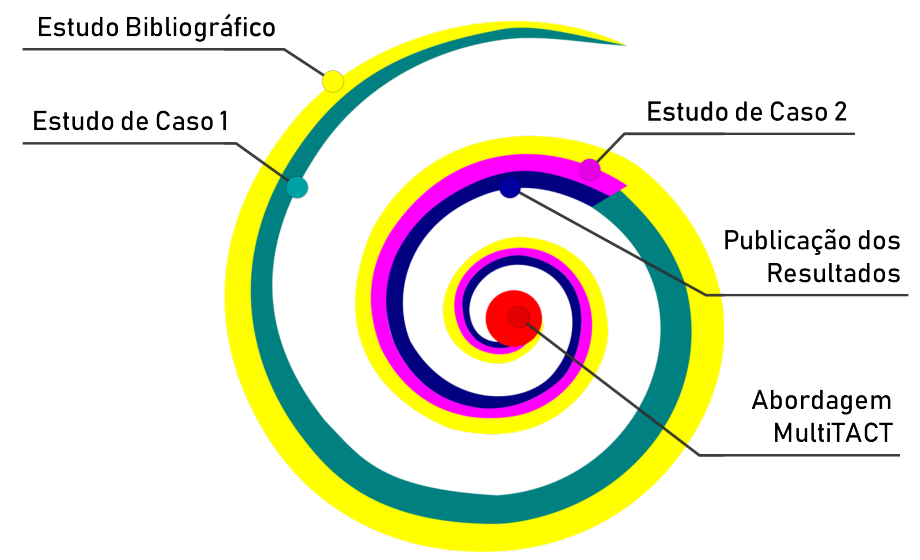

Figura 1. Visão geral da organização metodológica do estudo.

No Estudo Bibliográfico efetuou-se uma busca manual e automática [Gomes and Gomes 2019] a fim de levantar estudos sobre como o PC tem sido abordado no EFI e as abordagens existentes em torno do tema. A estratégia manual aconteceu diante da consulta de trabalhos publicados em conferências e periódicos nas áreas de Computação e Educação. Já a busca automática foi feita nos repositórios IEEE Xplore, ACM, ScienceDirect, nas bases nacionais do Periódicos CAPES e da Comissão Especial de Informática na Educação (CEIE). Aplicou-se strings com os termos Computacional Thinking, Primary School, Framework e suas traduções em Língua Portuguesa.

No Estudo de Caso $^{1} 1$ (EC1), de caráter exploratório, investigou-se sobre: a) como é possível incluir o PC em atividades de ensino, e b) como o docente trata/fomenta as habilidades do Pensamento Computacional em atividades de ensino. Para tanto, foi desenvolvida uma formação continuada na modalidade de curso de extensão presencial, a fim de que os participantes atuassem na construção e aplicação de práticas de ensino em classes do EFI. As práticas foram obtidas por meio dos artefatos de coleta nomeados como Planilha de Dados Primários (PAE) e Roteiro da Atividade de Ensino (RAE). Os docentes participavam de brainstormings para documentar no PAE elementos como a finalidade da atividade, habilidades do PC desenvolvidas, componentes curriculares relacionados e materiais. O RAE era preenchido após a condução da prática no EFI, sendo dedicado à descrição da atividade (metodologia) e à reflexão do docente sobre o pós prática. $\mathrm{O}$ curso foi realizado no primeiro semestre de 2017, em parceria entre a UFSCar Sorocaba e a Secretaria de Educação de Salto de Pirapora. As atividades obtidas e analisadas forneceram resultados que apoiaram decisões no estudo posterior.

No Estudo de Caso 2 (EC2), de caráter avaliativo, pesquisou-se: a) quais os elementos que sintetizam uma abordagem destinada ao desenvolvimento de atividades que fomentem o Pensamento Computacional, e b) como é identificada a relação das habilidades do PC e da BNCC em práticas de ensino. Foram feitas duas novas turmas de formação

\footnotetext{
${ }^{1}$ Método empírico que visa investigar um fenômeno em um dado contexto. Permite escolher múltiplos meios de coleta de dados para obter uma compreensão em profundidade do caso [Creswell 2014].
} 
VIII Congresso Brasileiro de Informática na Educação (CBIE 2019)

Anais dos Workshops do VIII Congresso Brasileiro de Informática na Educação (WCBIE 2019)

continuada como cursos de extensão, em formato semipresencial, que novamente possibilitou aos envolvidos efetuarem o planejamento e a aplicação de atividades direcionadas ao fomento do PC. No EC2 as atividades foram obtidas pelo Modelo de Atividade (MA). Os docentes documentaram no MA o objetivo da atividade, as habilidades do PC e da BNCC, entre outros campos usuais em um plano de aula. Após a condução da atividade no EFI, eram feitas alterações no próprio MA, caso necessário. Diante da técnica de Observação Não-Participante, algumas práticas foram acompanhadas pela primeira autora, com o intuito de capturar informações por meio de protocolos observacionais. Os cursos foram feitos no primeiro semestre de 2018, com uma turma ministrada na UFSCar Sorocaba e outra no IFSP Câmpus Itapetininga. As práticas obtidas e observadas agregaram novos dados para aprofundar a análise qualitativa.

Em todas as formações os docentes produziram práticas aplicáveis no EFI, capazes de estimular as habilidades do PC e da própria BNCC. A documentação dessas atividades e outros materiais (como fotos, vídeos e protocolos observacionais) foram submetidos a uma análise qualitativa, guiada pela Teoria Fundamentada (Grounded Theory) [Creswell 2014]. Na Teoria Fundamentada busca-se por conceitos (abstrações dos dados brutos), diante de sucessivas comparações entre os dados para formar uma teoria. Os processos de codificação da técnica podem ser definidos como: a) Codificação Inicial (Nivel 1): Extração de comportamentos, eventos, estados e condições observadas nos dados; identificação de conceitos emergentes, b) Codificação Axial (Nível 2): Sintetização dos códigos mais significativos e frequentes; desenvolvimento de comparações entre casos, e c) Codificação Teórica (Nivel 3): Interpretação descritiva dos dados que sustentem os eventos e interpretação que justifique como ou por que se dá a conexão entre categorias; inferência de categorias e temas que constituem a teoria proposta [Charmaz 2009].

Com uma análise guiada pela Teoria Fundamentada pôde-se conceber a Abordagem MultiTACT. O diferencial da metodologia adotada encontra-se em um estudo que partiu da prática, das atividades de ensino construídas pelos participantes e observadas, transformando-as em uma teoria. A Teoria Fundamentada tem sua importância por viabilizar uma abstração, fundada em dados derivados da prática docente, e que pode orientar outros professores que sentem dificuldades em criar atividades que fomentem o PC.

\section{Resultados dos Estudos de Caso}

São apresentados os principais resultados qualitativos que justificam a composição da Abordagem MultiTACT. As categorias obtidas e suas respectivas relações entre os primeiros conceitos extraídos da análise qualitativa, levaram à composição de três Formatos de Atividades de Ensino (FAEs). Tais estruturas resultaram da análise das 13 práticas capturadas no EC1. Nas FAEs, observou-se uma mudança no tratamento do PC, abordado tanto como método (meio de condução da atividade) como objetivo (finalidade / propósito).

Das teorias (FAEs) concebidas no EC1, o Formato de Atividade de Ensino 2 (FAE2) foi o resultado selecionado para passar por um refinamento diante dos processos da Teoria Fundamentada. A escolha do FAE2 (Figura 2) no aprofundamento da análise justifica-se por representar atividades que exploraram o Pensamento Computacional de maneira integrada a um Componente Curricular, desenvolvendo habilidades de ambos a partir do Objetivo estimado, como dado na intersecção do diagrama. No FAE2 o Pensamento Computacional é fomentado a partir de assuntos relacionados à Ciência 
VIII Congresso Brasileiro de Informática na Educação (CBIE 2019)

Anais dos Workshops do VIII Congresso Brasileiro de Informática na Educação (WCBIE 2019)

da Computação (como Representação de Imagens por Pixels ou Números Binários) e são aliados a Outros Meios de Condução, como a Gamificação e a Cultura Maker [Martinelli et al. 2018], representados pelas setas no diagrama.

Considerando o FAE2, além das outras 38 práticas de ensino obtidas no estudo de caso seguinte, pôde-se aprofundar nos processo de análise qualitativa dos dados durante o EC2. As sucessivas comparações levaram a um refinamento do FAE2, alcançando categorias mais abstratas e que fundamentam a teoria emergente do Formato de Atividade de Ensino Comprimido (FAEC). O FAEC apresenta a sintetização de uma abordagem voltada ao desenvolvimento de atividades de ensino que fomentem o Pensamento Computacional no EFI e fornece bases à composição da Abordagem MultiTACT.

No diagrama do FAEC (Figura 2) há uma intersecção entre as Habilidades do $P C$ e as Habilidades da BNCC, uma vez que as atividades analisadas desenvolveram, a partir de um Objetivo / Problema, habilidades do PC e da BNCC. As demais categorias, dadas por setas, que tratam da organização e mediação das atividades, fundamentadas como: a) Estratégias de Ensino - códigos como Objetos de Aprendizagem, Produção de Jogos, Cultura Maker e Assuntos de Computação identificados como mecanismos usados pelos docentes para conduzirem as práticas, b) Recursos e Materiais - códigos como materiais de baixo custo, produção de artefatos, softwares e aplicativos como alguns dos recursos utilizados junto das Estratégias de Ensino, e c) Ambiente e Contexto - códigos dos locais em que as práticas foram aplicadas, como sala de aula, ambientes externos e espaços maker, além das características associadas aos contextos de ensino promovidos pelas práticas, como ambientes desafiadores, motivadores e de criatividade.

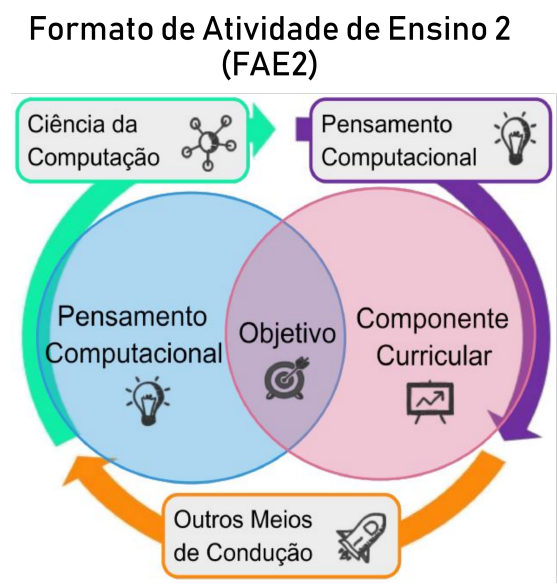

\section{Formato de Atividade de Ensino Comprimido} (FAEC)

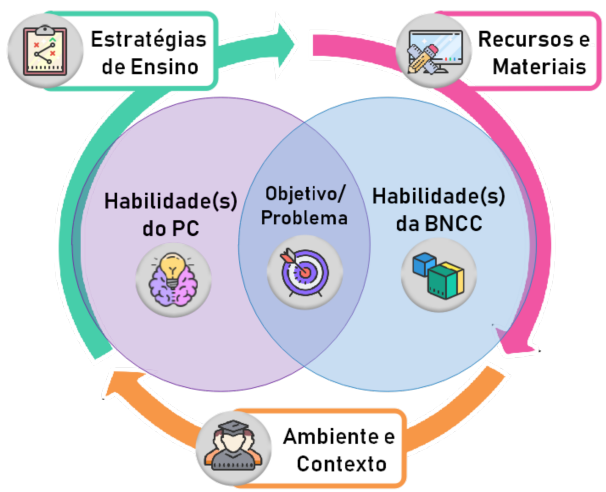

Figura 2. Resultados qualitativos obtidos durante o EC1 e o EC2.

Outros resultados são os códigos da Tabela 1 e da Tabela 2. Elas exibem as ações e processos mais frequentes identificados nas práticas, que se relacionam às habilidades da BNCC e foram estimuladas de maneira concomitante ao PC. Os códigos são organizados por sentenças, agrupados por área do conhecimento. Eles recebem uma sigla ${ }^{2}$ e um número sequencial. O cruzamento entre dados dá o valor percentual de citações igualmente presentes entre dois códigos [Charmaz 2009]. Das correlações entre os códigos da BNCC e do PC, alguns resultados identificados foram na área de Linguagens entre LP04 e Algoritmos / Procedimentos, com uma correlação de 9\%, enquanto que na Matemática

\footnotetext{
${ }^{2}$ Língua Portuguesa (LP), Artes (AR), Matemática (MA), Ciências (CI), Geografia (GE) e História (HI).
} 
VIII Congresso Brasileiro de Informática na Educação (CBIE 2019)

Anais dos Workshops do VIII Congresso Brasileiro de Informática na Educação (WCBIE 2019)

o MA06 apresentou 10\% de correlação com Decomposição / Generalização e Abstração. Houve também o fomento de habilidades da BNCC e do PC por temas como a produção e/ou interpretação colaborativa de texto (LPO4) e por estratégias de cálculo mental e estimativas (MA06). Outras correlações foram entre o CIO4 e a Abstração e entre o GE03 e a Manipulação de Dados, ambas com 6\% cada.

Tabela 1. Códigos identificados na análise qualitativa, respectivos à BNCC.

\begin{tabular}{|l|l|}
\hline \multicolumn{1}{|c|}{ Área de Linguagens } & \multicolumn{1}{c|}{ Área de Matemática } \\
(LP01) Reconhecer diferentes gêneros textuais. & $\begin{array}{l}\text { (MA01) Interpretação e manipulação de dados em } \\
\text { tabelas. } \\
\text { (MA02) Aplicação de medida padronizada. }\end{array}$ \\
(LP02) Reconhecer letras e/ou compor & $\begin{array}{l}\text { (MA03) Problemas sobre situações comerciais e } \\
\text { palavras. }\end{array}$ \\
$\begin{array}{l}\text { sistema monetário. } \\
\text { (MP03) Interpretação de linguagem verbal e } \\
\text { não verbal. }\end{array}$ & $\begin{array}{l}\text { (MA05) Aplicabilidade das operaçães fundamentais. } \\
\text { (MA06) Estratégias de cálculo mental e estimativa. }\end{array}$ \\
(LP04) Produção e/ou interpretação & (MA07) Quantificação, agrupamento e ordenação \\
colaborativa de texto. & $\begin{array}{l}\text { de objetos. } \\
\text { (MA08) Trajetos e pontos de origem / destino. }\end{array}$ \\
(AR01) Explorar brincadeiras regionais. & (MA09) Deslocamentos e mudanças de direção, \\
& sentido e giro. \\
(AR02) Uso de softwares e multimídias na & (MA10) Composição de formas geométricas e/ou \\
produção / criação artística. & análise de seus atributos. \\
(AR03) Uso de diferentes técnicas e materiais & (MA11) Solução de um mesmo problema com \\
na composição de artes visuais. & diferentes algoritmos. \\
\hline
\end{tabular}

Tabela 2. Códigos identificados na análise qualitativa, respectivos à BNCC.

\begin{tabular}{|c|c|}
\hline Ireza & Área de Ciências Humanas \\
\hline $\begin{array}{l}\text { (CI01) Identificar constelações. } \\
\text { (CI02) Reciclagem e identificação de } \\
\text { recicláveis. } \\
\text { (CI03) Plantas e etapas de plantio. } \\
\text { (CI04) Identificar e comparar propriedades } \\
\text { entre materiais / objetos. } \\
\text { (CI05) Construção de dispositivos óticos. }\end{array}$ & $\begin{array}{l}\text { (GE01) Aspectos sobre economia, processos de } \\
\text { produção e consumo. } \\
\text { (GE02) Pontos cardeais e de referência. } \\
\text { (GE03) Interpretação e/ou representação de } \\
\text { espaços e mapas. } \\
\text { (HI01) Papéis sociais das pessoas nas } \\
\text { comunidades. } \\
\text { (HI02) Comparação de diferentes linguagens e } \\
\text { seus aspectos culturais. }\end{array}$ \\
\hline
\end{tabular}

Observa-se que diferente do que é exposto pela BNCC, o PC é uma competência pertinente de ser desenvolvida em todas as áreas do conhecimento e não apenas na Matemática. Tal observação leva à inferência da multidisciplinaridade do PC, uma vez que este é estimulado a partir de vários componentes curriculares.

\section{A Abordagem MultiTACT: Fundamentos e Discussões}

As etapas que compõem a Abordagem MultiTACT são apresentadas por retângulos no fluxograma da Figura 3. Cada etapa determina um conjunto de conceitos ou ações a serem compreendidos(as), aplicados(as) e/ou documentados(as) para desenvolver atividades sobre PC. Por ser um método cíclico, há decisões, dadas por losangos, que possibilita ao professor escolher qual processo necessita ser efetuado ou refeito.

Discute-se aqui o emprego da abordagem em relação a todas as suas etapas. Assim, na fase Conhecer é necessário que o docente conheça ou seja apresentado ao PC e à $\mathrm{BNCC}$, bem como ao dados conceitos e/ou técnicas: a) Tipos de Atividades: pode ser Desplugada, Plugada (com o uso de aplicações e ferramentas digitais), ou ser Desplugada 


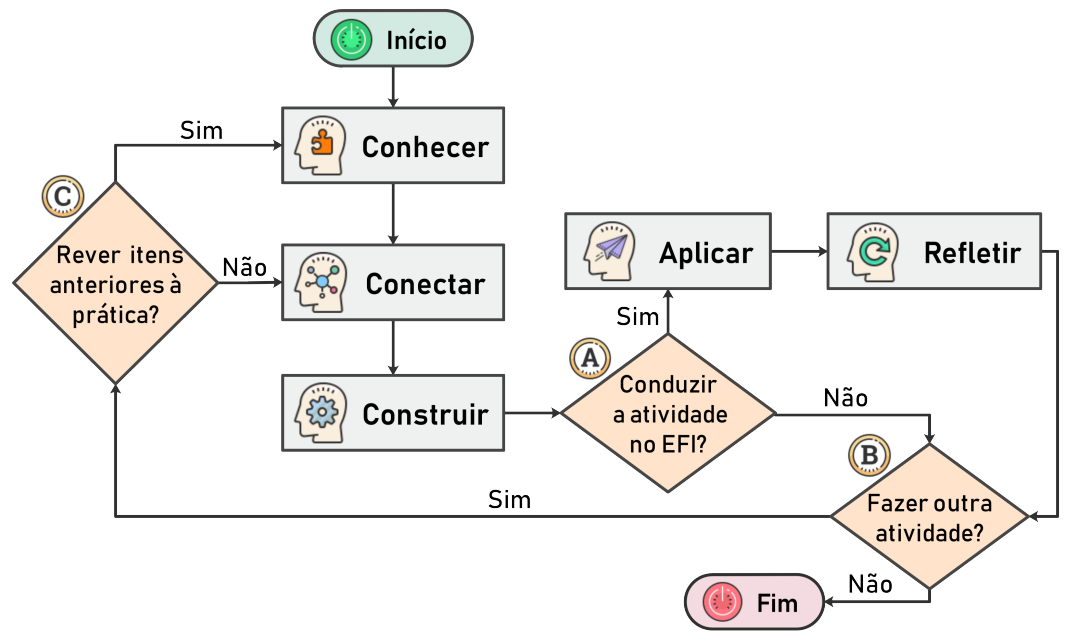

Figura 3. Fluxograma da Abordagem MultiTACT.

/ Plugada, que combina momentos de uso e não uso de meios digitais; b) Estratégias de Ensino: mecanismos de apoio à organização e mediação das práticas dedicadas ao estímulo do PC, reconhecidos como Aprendizagem Baseada em Problemas, Cultura Maker, Gamificação, Objetos de Aprendizagem, Produção de Animações e/ou Narrativas Digitais, Produção de Simulações e/ou Jogos Digitais, Programação Visual com Blocos Lógicos, Robótica Educacional e Temas/Assuntos sobre Computação ${ }^{3}$; e c) Aplicações, Software e Recursos: incluem o uso de materiais de baixo custo (como itens de papelaria, materiais recicláveis e componentes eletrônicos reutilizáveis) até a utilização de recursos digitais como jogos, simulações e linguagens baseada em blocos lógicos ${ }^{4}$. Sobre as Estratégias de Ensino, vale pontuar que as estratégias apresentadas foram incorporadas à abordagem por serem mecanismos detectados nas ações dos docentes ao elaborarem práticas dedicadas ao PC. Porém, a escolha por uma ou mais estratégias de ensino não se limitam aquelas recomendadas pela abordagem, sendo possível o docente elencar outros meios. A mesma lógica se aplica ao subgrupo de Aplicações, Softwares e Recursos.

Os conhecimentos anteriores influenciam na projeção de atividades na etapa $\boldsymbol{C o -}$ nectar. Nessa fase, o docente define um Objetivo / Problema a ser resolvido pelo discente, sendo essa problemática associada às habilidades do $\mathrm{PC}$ e da $\mathrm{BNCC}$, as quais se espera que sejam desenvolvidas pelos alunos ao resolverem o problema. A Figura 4 exibe os campos de preenchimento do Modelo de Planejamento Prévio e Conexões ${ }^{5}$ (MPPC). Ele oferece um instrumento de planejamento, centrado nos mesmos propósitos do FAEC. Após o preenchimento das unidades internas do MPPC, o professor define os Temas ou Conceitos Chaves a serem abordados, quais as Estratégias de Ensino a serem usadas, os Recursos e Materiais a serem aplicados e os Locais / Espaços para Condução da prática.

Na fase Construir o professor preocupa-se em documentar as ações sobre como o PC e a BNCC serão estimulados na condução da prática de ensino, junto dos recursos, estratégias e ambiente determinados. Para isso, o docente possui o Modelo de Plano de Aula $^{6}$ (MPA), que agrega campos para a especificação dos elementos definidos anteriormente, mas que podem sofrer alterações a partir da análise do docente ao compor o

\footnotetext{
${ }^{3}$ Definições das Estratégia de Ensino diante da Abordagem MultiTACT em: http://bit.ly/2PWVMZw.

${ }^{4}$ Sugestões de Recursos e Materiais diante da Abordagem MultiTACT em: http://bit.ly/2Yop5XT.

${ }^{5}$ Modelo de Planejamento Prévio e Conexões (MPPC) disponível em: http://bit.ly/2OfloW4.

${ }^{6}$ Modelo de Plano de Aula (MPA) disponível em: http://bit.ly/2YcaNJJ.
} 
VIII Congresso Brasileiro de Informática na Educação (CBIE 2019)

Anais dos Workshops do VIII Congresso Brasileiro de Informática na Educação (WCBIE 2019)

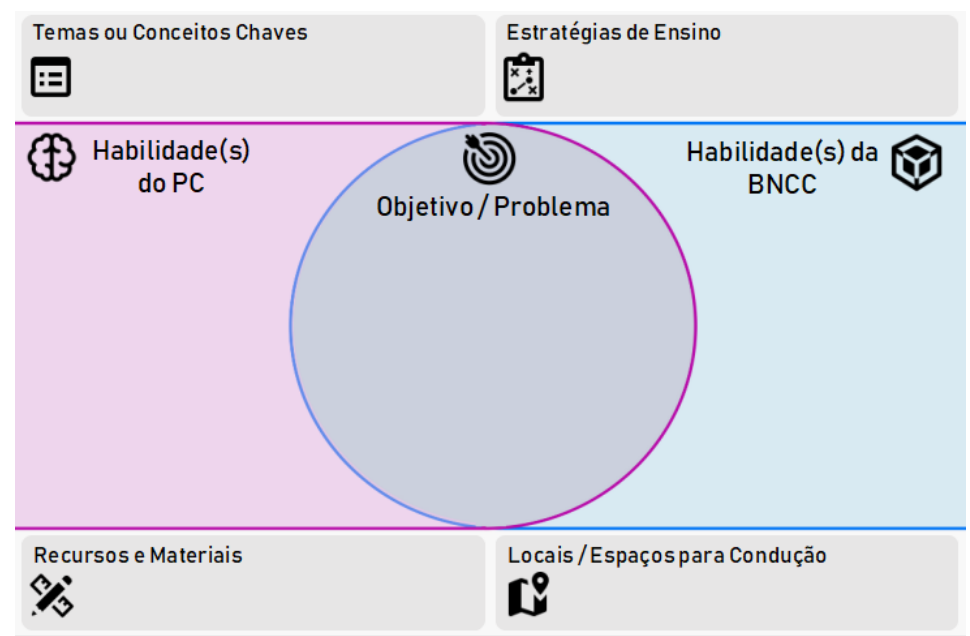

Figura 4. Representação do MPPC, pertinente à etapa Conectar.

plano de aula. O MPA é dividido em duas áreas de preenchimento, sendo a primeira pelo cabeçalho com informações curtas e de identificação da atividade, e a segunda, o corpo, para especificar as habilidades, o objetivos e o desenvolvimento passo a passo da prática.

Os diferenciais presentes no MPA são os campos Notas sobre Cenário / Ambiente, dedicado para descrever onde ocorrerá a atividade ou ilustrar como é o ambiente da prática, e o Observações e Sugestões de Adaptação elou Avaliação, reservado para marcar adaptações que podem ser aplicadas ao plano de aula desenvolvido. Além da construção do plano de aula com o MPA, a fase compreende a elaboração de artefatos ou Objetos de Aprendizagem que venham a ser usados na atividade e que foram anteriormente definidos.

A etapa Aplicar é reservada para a condução da atividade planejada em uma classe do EFI. Considerando a condução dessas dinâmicas e os atributos da Abordagem Multi$T A C T$, são fornecidas recomendações extraídas a partir das práticas analisadas. A fim de orientar os docentes no antes e durante a aplicação da atividade, têm-se como exemplo as práticas a seguir, sendo recomendado nessa etapa: a) avaliar o que foi sistematizado em Notas sobre o Cenário / Ambiente e nos Recursos e Materiais do MPA, pois pode ser necessário organizar com antecedência o cenário imaginado para a condução da prática (Foto A - Figura 5), b) testar com antecipação os softwares e/ou dispositivos digitais selecionados para uso (Foto B - Figura 5), e c) quando for possível, ter a participação de mais de adulto para colaborar na condução da atividade (Foto C - Figura 5).

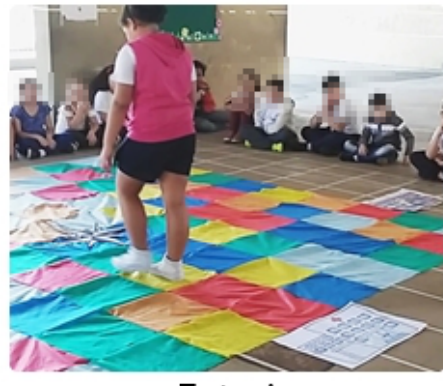

Foto $\mathrm{A}$

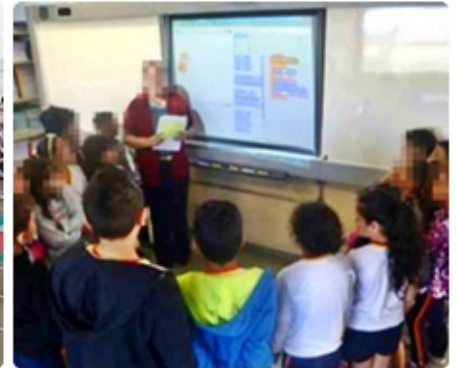

Foto B

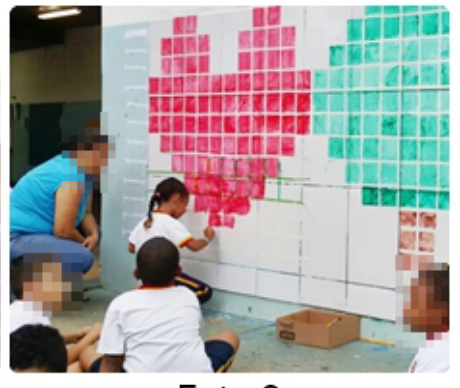

Foto C

Figura 5. Registros de diferentes atividades, relacionadas a etapa Aplicar.

E na etapa Refletir, o professor faz uma análise sobre a prática aplicada. Qua- 
VIII Congresso Brasileiro de Informática na Educação (CBIE 2019)

Anais dos Workshops do VIII Congresso Brasileiro de Informática na Educação (WCBIE 2019)

tro questionamentos devem ser considerados para reflexão: a) Os alunos conseguiram resolver o problema proposto ou atingir os objetivos? b) Foi possível estimular nos alunos as habilidades esperadas? c) Há itens que precisam de adequações na atividade? e d) Quais os impactos/consequências proporcionados(as) pela atividade pautada no PC? como atenção, colaboração, criatividade e motivação entre os alunos. Refletir sobre esses aspectos pode levar o docente à alterar ou adicionar descrições no MPPC e no MPA.

O envolvimento do docente em ações e processos da Abordagem MultiTACT estimulam uma práxis que permite conceber práticas educativas de múltiplas faces, sendo o PC abordado de maneira significativa ao currículo e contextualizado em diferentes problemas. O termo 'Múltiplas Facetas', presente no nome da abordagem, qualifica as atividades como abrangentes, flexíveis e multidisciplinares. A abrangência diz respeito às diferentes estratégias de ensino e materiais disponíveis ao docente para viabilizar atividades voltadas ao PC. A flexibilidade refere-se à possibilidade de adaptação das atividades em relação as estratégias, recursos e ambientes envolvidos, as quais são capazes de atender a um mesmo propósito ou objetivo, mas que possibilitam adequações diante da realidade institucional de cada professor. E a multidisciplinaridade caracteriza a articulação das habilidades do $\mathrm{PC}$ em assuntos relacionados aos diferentes componentes curriculares, o que viabiliza o desenvolvimento das habilidades dadas pela BNCC em justaposição com aquelas do PC.

A Abordagem MultiTACT reuniu atributos como a abrangência, a flexibilidade e a multidisciplinaridade, elementos não identificados de maneira conjunta em outras propostas. A teoria concebida fornece uma ferramenta que dá autonomia ao professor, em especial aqueles que não são formados em Computação, para compor situações-problemas que estimulem o PC em contextos que não se limitam à Matemática. A combinação teóricoprática da abordagem assume que o fomento ao PC requer compreensão das tecnologias existentes (o que usar), do conteúdo (o que ensinar) e da pedagogia (como ensinar).

\section{Considerações Finais}

A Abordagem MultiTACT traz contribuições importantes, ao tornar o docente o autor de atividades multidisciplinares sobre o PC e não apenas um coadjuvante que replica atividades prontas. Além da autoria, a abordagem viabiliza práticas pensadas a partir da realidade institucional do docente, projetadas de acordo com estratégias de ensino e materiais escolhidos. A concepção desta abordagem alinhada às necessidades dos docentes só foi possível através do uso da Teoria Fundamentada. Ao adotar este método pôde-se conceber uma abstração das atividades criadas pelos participantes, a fim de que tal proposta seja replicável ao cotidiano de outros professores. Sugere-se como forma de refinamento da Abordagem MultiTACT que ela seja avaliada por docentes do EFI, considerando todas as suas etapas e processos, durante uma nova formação continuada de oito encontros.

Nas contribuições acadêmicas têm-se dois artigos publicados no XXIV Workshop de Informática na Escola, que apresentam resultados parciais dos estudos de caso [Martinelli et al. 2018, Martinelli and Sakata 2018]. Nas contribuições sociais, as formações certificaram 62 professores que disseminaram o PC em seu cotidiano escolar. As intervenções propiciaram uma nova compreensão sobre esse "pensar computacional" e como estimulá-lo em sala de aula, uma vez que maioria dos participantes encaravam o PC como sendo o uso de Tecnologias Digitais de Informação e Comunicação na educação. Como trabalhos futuros, espera-se elaborar uma publicação com as atividades 
VIII Congresso Brasileiro de Informática na Educação (CBIE 2019)

Anais dos Workshops do VIII Congresso Brasileiro de Informática na Educação (WCBIE 2019)

obtidas neste estudo. As autoras agradecem o apoio financeiro dado pela Coordenação de Aperfeiçoamento de Pessoal de Nível Superior (CAPES) - código 001.

\section{Referências}

Charmaz, K. (2009). A construção da Teoria Fundamentada: Guia Prático para Análise Qualitativa. Bookman Editora, Porto Alegre - RS.

Creswell, J. W. (2014). Investigação Qualitativa e Projeto de Pesquisa: Escolhendo entre Cinco Abordagens. Penso Editora, Porto Alegre - RS, $3^{\text {a }}$ edition.

França, R. and Tedesco, P. (2015). Um modelo para a aprendizagem do pensamento computacional aliado à autorregulação. In Anais dos Workshops do Congresso Brasileiro de Informática na Educação, volume 4, pages 85-94.

Gomes, A. S. and Gomes, C. R. A. (2019). Classificação dos tipos de pesquisa em informática na educação. In Metodologia de Pesquisa em Informática na Educação: Concepção da Pesquisa, Porto Alegre - RS. Sociedade Brasileira de Computação.

Gomes, T. C. S. (2018). Desenvolvimento do pensamento computacional na educação infantil: Contribuições de uma pesquisa-ação educacional. Master's thesis, Universidade Federal de Pernambuco, Recife - PE.

INEP (2019). Notas estatísticas: Censo escolar 2018. Instituto Nacional de Estudos e Pesquisas Educacionais Anísio Teixeira (INEP). Brasília - DF.

Martinelli, S. and Sakata, T. (2018). A disseminação do pensamento computacional por docentes do ensino fundamental I: Relatos de experiências e discussões. In Anais do Workshop de Informática na Escola, volume 24, pages 235-244.

Martinelli, S., Zaina, L., and Sakata, T. (2018). O pensamento computacional em atividades de ensino mediadas pelo professor do ensino fundamental i: Um estudo de caso. In Anais do Workshop de Informática na Escola, volume 24, pages 509-518.

Mohaghegh, M. and Mccauley, M. (2016). Computational thinking: The skill set of the 21 st century. 7:1524-1530.

Nicastro, F., Baranauskas, C., and Torres, R. (2018). A methodology to conduct computational thinking activities in children's educational context. In Proceedings of the 10th International Conference on Computer Supported Education, pages 309-316.

SBC (2018). Ensino de computação na educação básica: Diretrizes para ensino de computação na educação básica. Sociedade Brasileira de Computação.

Seiter, L. and Foreman, B. (2013). Modeling the learning progressions of computational thinking of primary grade students. In Proceedings of the Ninth Annual International ACM Conference on International Computing Education Research, pages 59-66.

Sengupta, P., Kinnebrew, J. S., Basu, S., Biswas, G., and Clark, D. (2013). Integrating computational thinking with k-12 science education using agent-based computation: A theoretical framework. Education and Information Technologies, 18(2):351-380.

Valente, J. A., Freire, F. M. P., Arantes, F. L., Amiel, T., Baranauskas, M. C. C., et al. (2017). Alan turing tinha pensamento computacional? reflexões sobre um campo em construção. Tecnologias, Sociedade e Conhecimento, 4(1):7-22.

Wing, J. M. (2006). Computational thinking. Communications of the ACM, 49(3):33-35. 